\title{
Elastic Matching Using a Deformation Sphere
}

\author{
J. Lötjönen ${ }^{1}$ and T. Mäkelä2,3,4 \\ 1 VTT Information Technology, P.O.B. 1206, FIN-33101 Tampere, Finland \\ \{Jyrki.Lotjonen@vtt.fi\} \\ ${ }^{2}$ Laboratory of Biomedical Engineering, Helsinki University of Technology, P.O.B. 2200, \\ FIN-02015 HUT, Finland \\ 3 CREATIS, INSA, Batiment Blaise Pascal, 69621 Villeurbanne Cedex, France \\ ${ }^{4}$ BioMag Laboratory, Helsinki University Central Hospital, P.O.B. 503, \\ FIN-00029 HUCS, Finland
}

\begin{abstract}
A novel method is proposed for elastic matching of two data volumes. A combination of mutual information, gradient information and smoothness of transformation is used to guide the deformation of another of the volumes. The deformation is accomplished in a multiresolution process by spheres containing a vector field. Position and radius of the spheres are varied. The feasibility of the method is demonstrated in two cases: matching inter-patient MR images of the head and intra-patient cardiac MR and PET images.
\end{abstract}

\section{Introduction}

Proper interpretation and comparison of medical volumes from different modalities can be accomplished by transforming all data into common spatial alignment, also referred to as registration [1]. In many cases, a satisfactory solution can be found by using rigid registration, i.e. a volume is only translated and rotated. The registration algorithms can be coarsely divided into three groups which register: 1) a set of landmark points, such as external markers and anatomic landmarks [2], 2) geometric image features, such as edges [3], and 3) image intensity based similarity measures, such as mutual information (MI) [4,5]. Algorithms combining these groups exist too, e.g. Pluim et al. [6] used geometric features and intensity based similarity measures.

Elastic registration or matching is required as inter-patient volumes or regions containing non-rigid objects are registered. The goal is to remove structural variation between the two volumes to be registered. Many approaches have been proposed for the problem in recent years [7/8 9|10,11,12]. In the method proposed by Christensen et al. [7], physical properties of either elastic solids or viscous fluids were simulated as the model was deformed. The criterion for the deformation was to minimize the difference in voxel gray values between two volumes while constraining the transformation to be smooth. Wang and Staib [8] used intensity similarity combined with statistical shape information. The formulation of the elastic model was similar to the one used by Christensen et al. but the information on typical deformations, derived from individuals, was incorporated to guide the deformation. Thirion [9] developed a fast 3D matching method based on Maxwellian demons. The displacement vectors for the model were derived from an optical flow equation and smoothed by Gaussian filtering. MI was utilized in non-rigid registration by Gaens et al. [10]. In their approach, neighborhood 
regions around each point of a discrete lattice were locally translated so that MI was increased. Then, the calculated displacement, filtered by a Gaussian kernel, was applied to the points in the neighborhood. The process was iterated by decreasing gradually the size of the neighborhood and by using a multiresolution approach. Rueckert et al. [11] proposed a method where they applied MI and imposed the smoothness of the transformation to constrain the matching. The deformation of data was accomplished using a free-form deformation (FFD) deformation grid. Collins et al. [12] maximized a correlation between voxels in two volumes while simultaneously smoothing the transformation by the average displacement vector around each voxel of interest. FFD was used to deform the model. A multiresolution approach was applied.

If one volume to be registered is an atlas, i.e. a volume where the tissue classes of the voxels are known, the result of elastic matching provides also a segmentation. The use of elastic models or deformable models, such as snakes, in the segmentation is a widely studied field in medical image processing [13].

We propose a method by which a model volume with gray-scale information or an atlas consisting of gray-scale data and a set of triangulated surfaces is elastically matched to a data volume. In order to perform the registration, a weighted sum of three energy components is maximized. The first component is the MI between the images [4] while the second component is derived from the intensity gradients of the images [6]. The third component controls either the smoothness of the transformation [11] or the shape of the surfaces in the atlas [14]. The model is deformed using deformation spheres where the transformation is computed only for the model points inside the spheres. A high number of spheres with varying position and radius is used. In addition, a multiresolution approach is adopted.

\section{Methods}

In this study, the model used is a gray-scale volume taken from an individual. If the segmentation of the objects of interest is available, triangulated surfaces of these objects are incorporated in the model, i.e. the model is an atlas. In practice, the model contains also a gradient volume computed from the gray-scale data using a Canny-Deriche operator.

The multi-resolution approach is adopted. A low resolution volume is produced by Gaussian filtering and subsampling a high resolution volume. The matching is done first at the lowest resolution level. As the maximum energy is attained, the process is repeated for a higher resolution level.

Rigid registration is required before elastic transformation if the two volumes have significantly different initial positions. For reference, the mispositioning of the lungs by $5 \mathrm{~cm}$ was, however, recovered by the elastic matching in our tests.

In the following, the gray-scale volume of the model is referred to as a volume $M$, and the data volume taken from a patient, and to which the model is matched, volume $D$. Consequently, a sample point, i.e. a voxel, from the volume $M$ is $\mathbf{m}=\left(m_{1}, m_{2}, m_{3}\right)$ and from the volume $D \mathbf{d}=\left(d_{1}, d_{2}, d_{3}\right)$. The transformation applied to the sample points in the model volume is denoted by $\mathbf{T}:(x, y, z) \mapsto\left(x^{\prime}, y^{\prime}, z^{\prime}\right)$. 


\subsection{Energy Function}

The motivation in using more than one energy term is to create an energy function with less local minima and therefore to make matching more robust [6]. The energy components provide complementary information on the matching: the gradient component incorporates spatial information while the regularization of the transformation aims to preserve, in a way, the prior knowledge of the shape of the object.

Mutual information. MI measures the degree of dependence between the volumes $M$ and $D$. MI is high if the gray-scale value of the voxel $i$ in $D$ can be estimated with a high accuracy as the gray-scale value of the corresponding voxel in $M$ is known. If the gray-scale values of the volumes $M$ and $D$ are considered random variables $A$ and $B$, respectively, the MI, proposed in [4] and denoted here by energy $E_{M I}$, is be computed from the equation:

$$
E_{M I}=\sum_{a, b} p_{A B}(a, b) \log \frac{p_{A B}(a, b)}{p_{A}(a) p_{B}(b)},
$$

where $p_{A}(a)$ and $p_{B}(b)$ are marginal probabilites and $p_{A B}(a, b)$ is the joint probability distribution. $p_{A}(a)$ is the probability that the gray-scale value of a voxel is $a$ in volume $M . p_{A B}(a, b)$ is the probability that the corresponding voxels in the volumes $M$ and $D$ have the gray-scale values $a$ and $b$.

Joint gradient information. The points in the model should match similarly oriented points in the data. The method used is a simplified version of [6]. The energy component $E_{\text {grad }}$ derived from the gradients is computed as follows:

$$
E_{\text {grad }}=\frac{1}{N} \sum_{(\mathbf{m}, \mathbf{d}) \in(M \cap D)} \frac{\nabla \mathbf{m} \cdot \nabla \mathbf{d}}{|\nabla \mathbf{m}||\nabla \mathbf{d}|} \min (|\nabla \mathbf{m}|,|\nabla \mathbf{d}|) .
$$

where $N$ is the number of model points overlapping the volume $D$. Because a minimum of gradients is used in two volumes, the intensity ranges need to be set nearly similar in the both volumes.

Since the gray-scale value of a tissue depends on imaging sequence or imaging modality, the gradients on the edges of the tissue may have opposite directions in different volumes. If this is the case with volumes $M$ and $D$, Eq. 2 is modified by taking an absolute value from the dot-product.

Regularization of transformation. The transformation $\mathbf{T}$ can be constrained to be smooth by incorporating the energy component $E_{\text {model }, 1}[11]$ :

$$
E_{\text {model }, 1}=\frac{1}{N} \sum_{x, y, z}\left[\left(\frac{\partial^{2} \mathbf{T}}{\partial x^{2}}\right)^{2}+\left(\frac{\partial^{2} \mathbf{T}}{\partial y^{2}}\right)^{2}+\left(\frac{\partial^{2} \mathbf{T}}{\partial z^{2}}\right)^{2}+2\left(\frac{\partial^{2} \mathbf{T}}{\partial x \partial y}\right)^{2}+2\left(\frac{\partial^{2} \mathbf{T}}{\partial x \partial z}\right)^{2}+2\left(\frac{\partial^{2} \mathbf{T}}{\partial y \partial z}\right)^{2}\right]
$$

where the sum is over all voxels in the model volume and $N$ is the number of points summed. The energy term is the $3 \mathrm{D}$ counterpart of the $2 \mathrm{D}$ bending energy associated to a thin-plate metal. To speed up computations only three first terms are used in practice.

Alternatively, if the surface model is available, the smoothness of the transformation can be controlled by constraining the change in the shape of the model surfaces. In our 
study [14] where a boundary element template was matched to volume data, various regularization strategies were tested. The method with the aim of preserving the orientation of the model's surface normals was preferred. This method is applied also in this study. The energy component is computed as follows:

$$
E_{\text {model }, 2}=\frac{1}{N_{t r}} \sum_{i=1}^{N_{t r}} \mathbf{n}_{i} \cdot \mathbf{n}_{i}^{o}
$$

where $N_{t r}$ is the total number of triangles in the model, $\mathbf{n}_{i}$ and $\mathbf{n}_{i}^{o}$ are the deformed and the original directions of the normal of the triangle $i$, respectively.

Total energy. The model is deformed by maximizing the following energy function:

$$
E_{\text {total }}=E_{M I}+\alpha E_{\text {grad }}+\beta E_{\text {model }},
$$

where $\alpha$ and $\beta$ are user-defined weight parameters for the energy components.

\subsection{Model Deformation}

In our earlier study [14], a boundary element template was deformed using a FFD grid. Since the relative positions of the control points and the model points can not be arbitrarily chosen, the opportunity to control the transformation is limited. In this work, a volumetric transformation is used but the transformation can be better focused on the regions of interest. The potential of the method to include statistical shape information on the deformation is discussed in Section 4.

All model points, including voxels and surface points, inside a deformation sphere are transformed. The center $c=\left(c_{1}, c_{2}, c_{3}\right)$ and the radius $r$ of the sphere can be freely chosen. In practice, a high number of spheres (tens of thousands) are applied sequentially. The transformation vector $\mathbf{v}$ for a model point at $(x, y, z)$ inside the sphere is computed as follows:

$$
\mathbf{v}=\frac{e^{-k \frac{\left(x-c_{1}\right)^{2}+\left(y-c_{2}\right)^{2}+\left(z-c_{3}\right)^{2}}{r^{2}}}-e^{-k}}{1.0-e^{-k}} \mathbf{V},
$$

where $\mathbf{V}$ is a movement vector posed to the center of the sphere and $k$ is a parameter which specifies the sharpness of the weight function. A $2 \mathrm{D}$ version of the weight function with $k=3$ is visualized in Fig. 1.

The vector $\mathbf{V}$ is chosen in such a way that it maximizes the energy in Eq. 5. In practice, the center of the sphere is displaced to several locations, the model is deformed and energy computed, and the location having the highest energy is chosen. The user can define the number of locations tested $\left(N_{l o c}\right)$ around the center as well as the maximum displacement for the center $(s)$, e.g. $s=0.3 r$. The six closest neighbors in $3 \mathrm{D}$ are always included in the set of tested locations. In Fig. 1,b, the grid around the center of the sphere, shown by a circle in $2 \mathrm{D}$, represents the search space and visualizes the possible new locations for the center. The locations tested are shown by gray squares and the center by a black square. The motivation for testing several locations is that the simple gradient descent method is known to attach relatively easily to local energy minima or maxima. In this strategy, the energy function is maximized more globally. 


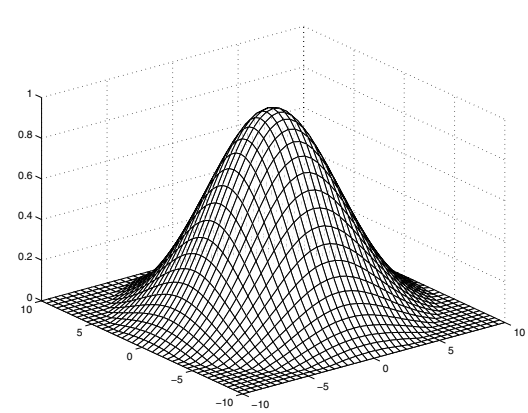

(a)

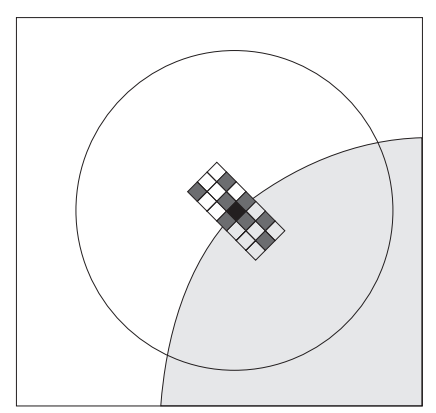

(b)

Fig. 1. (a) A $2 D$ weight function for the deformation $(r=10)$. (b) A $2 D$ search space visualized in the center of the sphere $\left(N_{l o c}=8\right)$.

By default, the maximum displacement $s$ is equal in all directions. In other words, the search space is cubic. However, many methods applying deformable models prefer deformations in the direction of the surface normals. This approach can be followed, if the surface is available in the model. The approach is accomplished as follows. First, define all surface points inside the sphere and compute the average of the surface normals by weighting each normal according to the weight in Eq. 6 . Denote the length of the average vector by $q$. Then, define $s=s$ in the direction of the average and $s=(1-q) s$ in orthogonal directions. In Fig. 1 b the surface is represented in 2D by the contour around the light gray object. Since the average of the surface normals inside the sphere is approximately in the diagonal direction, the value of $q \approx 1$. Therefore, the search space is highly anisotropic and the diagonal displacements are privileged.

By default, the locations of the spheres are randomly chosen inside the model volume $M$. However, if the surface information is included in the model, the locations are randomly chosen at the positions of the surface points. The model should contain surfaces for the regions which are required to be well matched in the final result. For example, if the lung borders are to be segmented from the image, the transformation inside and outside the borders are not of great interest. The use of the surfaces locates the deformation to the most interesting regions, and it speeds up the process because a great part of the volume is excluded, such as background. However, the spheres used at the lowest resolutions levels contain usually the whole or the most of the model, and allow therefore global transformation also for the regions far from the edges.

In the beginning of the deformation, the radius of the spheres is high. As the energy does not change more than a user-specified limit $\epsilon$ during an iteration, the radius of the sphere is reduced. The user can set the maximum and minimum radius. The number of spheres used in one iteration is relative to the volume of the model divided by the volume of the sphere. 


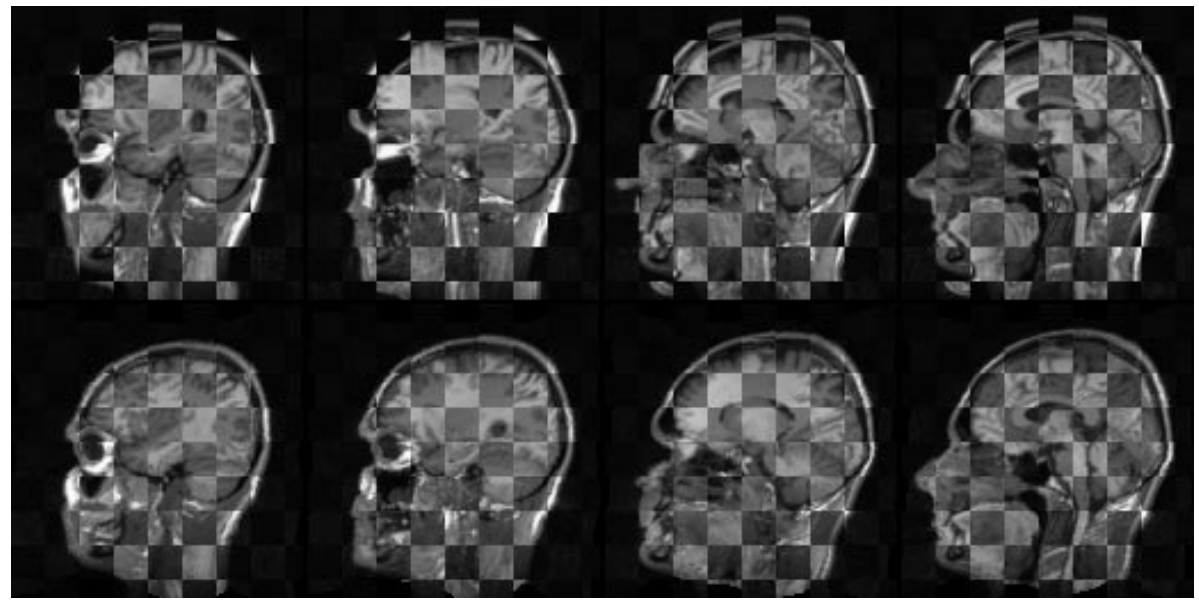

Fig. 2. Matching of two Tl-weighted MR volumes from the head. The top row shows the model and patient data interlaced before matching and the bottom row after elastic matching.

\section{Results}

The method was developed for two purposes. 1) An automated method was needed by which individualized geometric models can be built for bioelectromagnetic inverse problems from magnetic resonance (MR) images. 2) Elastic matching of cardiac MR and positron emission tomography (PET) images taken from a patient was also needed.

In Fig. 2, the top row shows the original model and patient data interlaced using a chessboard visualization. The bottom row presents the same volumes after the model was elastically matched with the patient data. By visual inspection the edges of anatomic objects appear well aligned after elastic matching, i.e. edges are reasonably continuous between the chess-boxes. The typical run time of the program is 5-15 minutes using a $600 \mathrm{MHz}$ Pentium as the size of the volumes is about $128 \times 128 \times 100$. In this case, the surface information was available and four resolution levels were used. The weights used in Eq. [5]were $\alpha=2$ and $\beta=10$. However, the result does not change appreciably as the weight values several times higher or lower are used. The radius of deformation spheres varied from 20 to 6 voxels.

Fig. 3 visualizes a result for cardiac MR-PET matching as the MR volume was the model. The top row shows the volume interlaced before elastic matching and the bottom row after matching. The left ventricle is matched reasonably well, e.g. the matching of the septum is indicated by the white arrows. In this case, the images were not rigidly registered before elastic matching. As the rigid registration is used, the images appear often well aligned already before the elastic transformation. The accurate assessment of the result is difficult because of highly blurred PET images. Therefore, the conditions for the use of elastic matching should be carefully evaluated in future studies. 


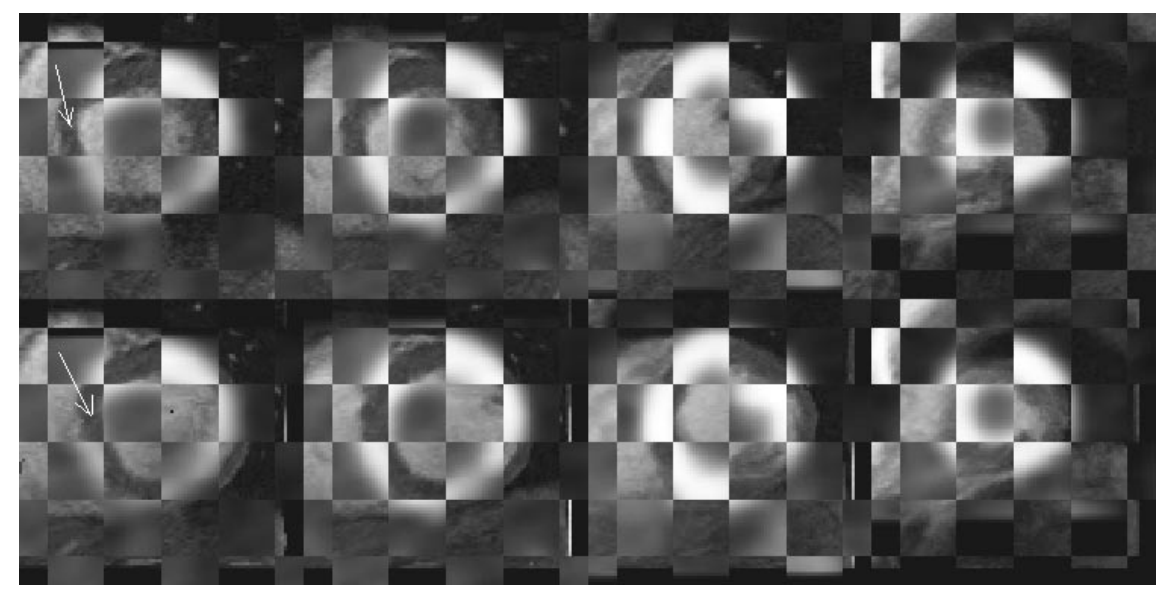

Fig. 3. Matching of cardiac MR and PET volumes from the left ventricle. The top row shows the model and patient data interlaced before elastic matching and the bottom row after matching.

\section{Discussion}

Elastic matching and segmentation of data volumes is often a pre-requisite for a successful diagnosis and treatment of a patient. This work proposes a method by which this image processing problem can be solved with a reasonable accuracy in a few minutes. So far, the method has been, however, tested only for a few volumes and the results are therefore preliminary.

Although the program allows several parameters to tune the matching, the values do not need to be often varied in practice. Proper parameter values for the energy components depend on imaging modality. If intra-modality anatomic images, such as MRI or computerized tomography (CT), are used, gradient information is weighted more in Eq. 5. However, mutual information should have a major impact on images which have very smooth gradients, such as PET images. The more pathologic the images are, i.e. the images to be matched are highly different or noisy, the more the transformation should be regulated.

Two methods to compute $E_{\text {model }}$ was presented. If a surface is not included in the model, only the smoothness of the transformation can be controlled. If the surface is available, the method regulating the normal directions is recommended, because it makes the run time remarkably shorter.

The method will be further improved by including statistical shape information. This can be done by defining typical transformations for various positions of the model. In the current version of the method, the deformations in the direction of the surface normals are preferred. However, information could be incorporated from test data for which the typical transformations are known, and constrain deformations to typical orientations. In the current version, all transformation vectors inside the sphere are parallel. Typical transformations could be better simulated, if curved transformations inside the 
sphere were applied. In addition, more general shapes than a sphere could be used to bound deformation regions, such as a "banana-shaped" object. This would allow constraining the deformation to a more specific region if necessary.

\section{Acknowledgements}

The authors express thanks to The Department of Radiology, Helsinki University Central Hospital, Finland, INSERM Unité 280, Lyon, France, and Turku PET Centre, Turku, Finland for providing volume images. The authors thank also Dr Patrick Clarysse for his constructive comments concerning the manuscript. Research was supported by the National Technology Agency, Finland.

\section{References}

1. J.B.A. Maintz and M.A. Viergever. A Survey of Medical Image Registration. Med. Image Anal., 2(1):1-36, 1998.

2. K.S. Arun, T.S. Huang and S.D. Blostein. Least-Squares Fitting of Two 3-D Point Sets. IEEE Trans. Pattern Anal. Machine Intell., 9(5):698-700, 1987.

3. P.A. Van den Elsen, J.B.A. Maintz, E-J.D. Pol and M.A. Viergever. Automatic Registration of CT and MR Brain Images Using Correlation of Geometrical Features. IEEE Trans. Med. Imag., 14(2):384-396, 1995.

4. F. Maes, A. Collignon, D. Vandermeulen, G. Marchal and P. Suetens. Multimodality Image Registration by Maximization of Mutual Information. IEEE Trans. Med. Imag., 16(2):187198, 1997.

5. P. Viola and W.M. Wells III. Alignment by Maximization of Mutual Information. Int. J. Comp. Vision, 24(2):137-154, 1997.

6. J.P.W. Pluim, J.B.A. Maintz and M.A. Viergever. Image Registration by Maximization of Combined Mutual Information and Gradient Information. IEEE Trans. Med. Imag., 19(8):809-814, 2000.

7. G.E. Christensen, M.I. Miller, M.W. Vannier and U. Grenander. Individualizing Neuroanatomical Atlases Using a Massively Parallel Computer. IEEE Computer, January, 1996.

8. Y. Wang and L.H. Staib. Elastic Model Based Non-rigid Registration Incorporating Statistical Shape Information. Lecture Notes in Computer Science 1496: Medical Image Computing and Computer-Assisted Intervention, MICCAI98, editors W.M. Wells, A. Colchester and S. Delp, Springer, 1162-1173, 1998.

9. J-P. Thirion. Fast Non-Rigid Matching of 3D Medical Images. INRIA Report 2547, 1995.

10. T. Gaens, F. Maes, D. Vandermeulen and P. Suetens. Lecture Notes in Computer Science 1496: Medical Image Computing and Computer-Assisted Intervention, MICCAI98, editors W.M. Wells, A. Colchester and S. Delp, Springer, 1099-1106, 1998.

11. D. Rueckert, L.I. Sonoda, C. Hayes, D.L.G. Hill, M.O. Leach and D.J. Hawkes. Nonrigid Registration Using Free-Form Deformations: Application to Breast MR Images. IEEE Trans. Med. Imag., 18(8):712-721, 1999.

12. D.L. Collins, C.J. Holmes, T.M. Peters and A.C. Evans. Automatic 3-D Model-Based Neuroanatomical Segmentation. Human Brain Mapping, 3:190-208, 1995.

13. T. McInerney and D. Terzopoulos. Deformable Models in Medical Image Analysis: a Survey. Med. Image Anal., 1(2):91-108, 1996.

14. J. Lötjönen, P-J. Reissman, I. E. Magnin and T. Katila. Model Extraction from Magnetic Resonance Volume Data Using the Deformable Pyramid. Med. Image Anal., 3(4):387-406, 1999. 